\title{
Fluorine as a Control Element in Asymmetric Synthesis
}

\author{
Vincent Bizet and Dominique Cahard*
}

\begin{abstract}
The installation of fluorine atoms in reactants or catalysts has a dramatic impact on reactivity, regioselectivity and stereoselectivity. Several effects account for the modified stereochemical outcome of reactions with fluorinated versus non-fluorinated molecules. These effects are inherent to the specific properties of fluorine such as the size, the electronegativity, the chelation with metal cations, the hydrogen-bonding ability, the electrostatic and stereoelectronic interactions with neighbouring groups. The use of the effects of fluorine for a desirable goal in asymmetric synthesis is exemplified hereafter.
\end{abstract}

Keywords: Asymmetric synthesis · Catalysis · Chirality · Fluorine · Gauche effect

Organofluorine chemistry was born from the combination of organic compounds derived from carbon and fluorine compounds originating from fluorspar $\mathrm{CaF}_{2}$. The two commodity chemicals hydrofluoric acid (HF) and elemental fluorine $\left(\mathrm{F}_{2}\right)$ are used to produce countless fluorinated organic molecules for numerous applications of daily life. From these raw materials, very sophisticated organofluorine compounds were elaborated including chiral optically pure molecules. ${ }^{[1]}$ Since the beginning of the new millennium, fluorine is undeniably the element that has experienced the strongest interest and it is not surprising to see that the literature abounds with efficient synthetic methods for the asymmetric construction of chiral fluorinated molecules. ${ }^{[2-4]}$ The preparation of these compounds can be achieved from readily available fluorinated building blocks early in a synthetic plan or by direct introduction of a fluorine atom or a fluorinated motif at any stage of a process. For this latter purpose, electrophilic, nucleophilic, and radical sources of fluorine or fluorinated groups can be used. Methods for diastereo- and enantiocontrolled $\mathrm{C}-\mathrm{F}$ bond formation through electrophilic $\alpha$-fluorination of carbonyl

\footnotetext{
${ }^{\star}$ Correspondence: Dr. D. Cahard

UMR 6014 CNRS COBRA

Normandie Université

1 rue Tesnière, 76821 Mont Saint Aignan, France

Tel.: +33235522466
}

E-mail: dominique.cahard@univ-rouen.fr compounds and derivatives as well as the fluorination of olefinic compounds have been efficiently developed thanks to the design of novel N-F reagents. Asymmetric nucleophilic fluorinations, which require fluoride sources (HF-amine complexes, $\mathrm{AgF}, \mathrm{PhCOF}$ ), have been less developed and are so far limited to the ring opening of meso-epoxides or aziridines, and reactions with allyl-Pd intermediates. For C$\mathrm{CF}_{3}$ bond formation, the nucleophilic trifluoromethylation extensively makes use of the Ruppert-Prakash reagent $\mathrm{Me}_{3} \mathrm{SiCF}_{3}$, while electrophilic trifluoromethylation including the use of the electrophilic $\mathrm{CF}_{3}$ radical has been developed thanks to the availability of Umemoto, Togni, Langlois, and Shibata $\mathrm{CF}_{3}$ donors. Concerning the $\mathrm{C}-\mathrm{R}^{\mathrm{f}}$ bond formation $\left(\mathrm{R}^{\mathrm{f}}\right.$ being a poly- or perfluorinated carbon chain with more than one carbon atom), this chemistry relies on the development of reagents similar to those used for the trifluoromethylation. The building block approach nicely complements the toolbox towards chiral fluorinated molecules; however, the methods developed for non-fluorinated reactants are not often easily transposed to fluorinated ones because of specific fluorine effects. Overall, highly important achievements have been reported in asymmetric synthesis of fluorinated compounds thanks to extensive research efforts towards ever higher yields and optical purities. The effect of fluorine on the reaction outcome can be dramatic in altering the reactivity, the regioselectivity, and the stereoselectivity. ${ }^{[5]}$ Indeed, fluorine has the potential to induce the selective formation of stereogenic centres through steric, electronic, and conformational effects. The judicious positioning of fluorine atom(s) on reactants, either the substrate or the catalyst, is a relevant tool in organic chemistry and in particular in asymmetric catalysis. Herein, we showcase selected examples of asymmetric reactions in which fluorine behaves as a control element.

\section{A Cumulative Size Effect}

The fluorine atom has a van der Waals radius of $1.47 \AA$ compared to $1.20 \AA$ for hydrogen and $1.52 \AA$ for oxygen. Although $22 \%$ bigger than hydrogen, fluorine, better than any other atom, causes minimal steric alteration when it replaces hydrogen. By cumulating fluorine atoms, as in the case of the trifluoromethyl group, makes this group much larger than the corresponding methyl group. The van der Waals molar volume of the $\mathrm{CF}_{3}$ group was calculated to be $21.3 \mathrm{~cm}^{3}$ $\mathrm{mol}^{-1}$, similar to the molar volume of the isopropyl group. Fluorine has undeniably a key role to play in reactions that involve size differentiation. The enantioselective reduction of prochiral ketones catalysed by (-)-B-chlorodiisopinocamphenylborane $\left[(-)-D I P-C h l o r i d e^{\mathrm{TM}}\right]$ is an illustration of the size effect of fluorine (Scheme 1). Brown, Ramachandran and co-workers observed a reversed enantiofacial selectivity during the reduction of non-fluorinated and fluoroalkylated ketones. ${ }^{[6,7]}$ Reduction of acetophenone led to the corresponding $(S)$ alcohol with $98 \%$ ee thanks to a sufficiently large size difference between the phenyl and the methyl substituents of the ketone. Mono-, and difluoromethyl groups, $\mathrm{CH}_{2} \mathrm{~F}$ and $\mathrm{CHF}_{2}$, displayed the same enantiofacial selectivity observed for acetophenone and gave the corresponding $(R)$ alcohols in $95 \%$ and $85 \%$ ee, respectively. Due to CIP stereochemistry rules, the absolute configuration is opposite but the reduction did occur on the same face for acetophenone and its two fluorinated analogues. The decrease in $e e$ values can be explained by the increasing size of the fluoroalkyl 
group $\mathrm{CH}_{2} \mathrm{~F}$ and $\mathrm{CHF}_{2}$ that caused a smaller size difference between the large and the small groups of the ketone and thus an attenuated enantioface discrimination. A methyl to $\mathrm{CF}_{3}$ substitution significantly increased the steric bulk and the reduction of 2,2,2-trifluoroacetophenone unexpectedly gave the $(S)$ isomer with $90 \%$ ee. In this case, the $\mathrm{CF}_{3}$ group acted as the enantiocontrolling larger group as compared to the phenyl group placing the phenyl group in the opposite position in the favoured transition-state (Scheme 1). The $\mathrm{CF}_{3}$ and the phenyl groups have indeed different shapes and flexibilities but it would mean that the volume of the $\mathrm{CF}_{3}$ group would be bigger than the phenyl group. To further demonstrate that steric factors governed the stereochemical outcome of these reactions, the pivalophenone substrate having a $t$-Bu group gave the $(R)$ enantiomer by reduction. The $t$-Bu group in the favoured transition-state was placed in the same position than the $\mathrm{CF}_{3}$ group on 2,2,2-trifluoroacetophenone. These examples clearly illustrated the size effect of fluorine that controlled the face selectivity in carbonyl reduction of prochiral ketones. The use of other chiral reducing agents gave divergent results; for instance, the structurally related (-)- $B$-isopinocamphenyl9-borabicyclo[3.3.1]nonane [R-AlpineBorane] gave the same sense of asymmetric induction for tri-, di- and monofluoroacetophenone albeit in different $e e$ values, 32, 97 and $89 \%$, respectively. A general trend was difficult to predict and a possible chelating effect of the fluorine and the strong Lewis acidic boron atom in the borane-ketone charge-dipole complex could not be completely ruled out to rationalise these experimental observations.

\section{A Chelation Effect with Metal Cations}

The fluorine atom is able to induce conformational control in cyclic intermediates through interaction with cations. Yamazaki and co-workers demonstrated that, in the diastereoselective alkylation of $\alpha$-alkoxyesters, the presence of at least one fluorine atom was responsible for a considerable improvement in the diastereoselection from 2-56\% to up to $90 \%$ de (Scheme 2). ${ }^{[8]}$ Deprotonation of the substrate by KHMDS afforded the potassium enolate with a frozen $Z$ configuration caused by an intramolecular chelation with the $\alpha$-oxygen atom and further formed a bicyclo [3.3.0]-type structure through intramolecular potassium...fluorine interactions. $A b$ initio computation of a model lithium enolate indicated a $\mathrm{Li} \cdots \mathrm{F}$ distance of 1.879 $\AA, 0.043 \AA$ shorter than the $\mathrm{Li}$...O distance, and a concomitant elongation of the $\mathrm{C}-\mathrm{F}$

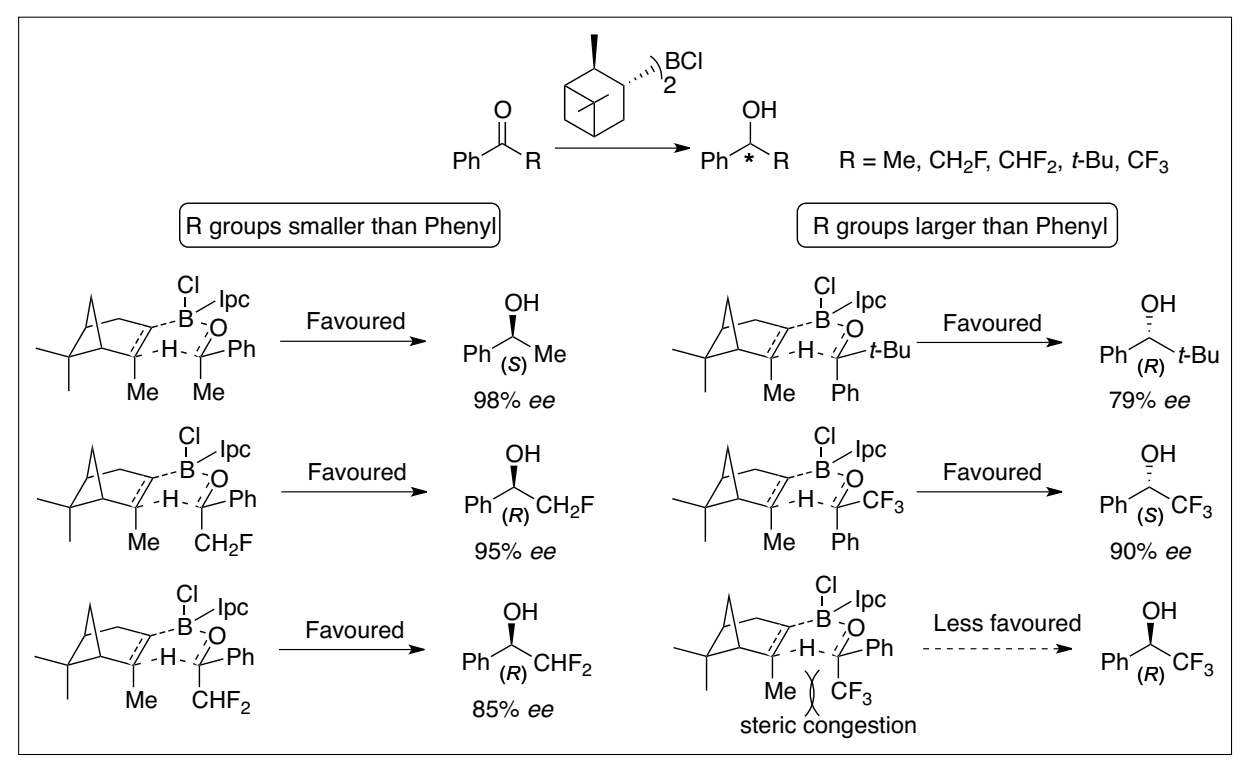

Scheme 1. Reverse sense of enantioselectivity caused by steric effect. bond $(0.072-0.078 \AA)$. The addition of the electrophile occurred preferentially at the more accessible convex face of the enolate (Scheme 2). The size of the cation was revealed to be crucial for the reactivity and a potassium atom appeared necessary to get high yields. In this reaction, the fluorine atom had minimum steric effect but imparted excellent discrimination of the enolate faces thus leading to significantly improved diastereoselectivities relative to the use of non-fluorinated substrates. The number of fluorine atoms was also investigated and gave diastereoisomeric excesses in the same range for $\mathrm{CH}_{2} \mathrm{~F}, \mathrm{CHF}_{2}$ and $\mathrm{CF}_{3}$ groups. The same group also reported that a metal $\cdots$ fluorine interaction was also operative in a transitory cyclic species during the alkylation of glycinate substrates. ${ }^{[9]}$

\section{Intermolecular Hydrogen-bonding Effect}

Fluorocarbons can act as hydrogen bond acceptors although this interaction is still the subject of open debate. This weak interaction is difficult to identify by experimental methods but can be more easily investigated by DFT calculations. In the organocatalytic asymmetric Strecker reaction of difluoro- and trifluoromethyl keto- imines, Wang, Zhou and co-workers have evidenced a remarkable fluorine effect suggesting a recognition model based on hydrogen-bonding interactions. ${ }^{[10]}$ The authors speculated that fluorine atoms of the ketoimines would form hydrogen-bonded intermediates with the urea hydrogens of the organocatalysts, an hypothesis that was underpinned by DFT calculations. Indeed, calculated optimised structures showed that the urea established hydrogen bonds with the imine nitrogen of fluorinated ketoimines $\mathrm{N} \cdots \mathrm{H}-\mathrm{N}$ and with a fluorine atom C-F...H-N (Scheme 3). The bifunctional chiral urea derivative of quinine, which was used in this study, featured a Brønsted acid moiety for $\mathrm{H}$-bonding interactions with the imine and a Lewis base moiety to activate the nucleophile trimethylsilyl cyanide (TMSCN). When the methylketoimine was reacted with TMSCN in the presence of the chiral catalyst, the expected product was obtained in high yield but as a racemic mixture. In this case, the non-fluorinated ketoimine formed two $\mathrm{H}$-bonds with both urea hydrogens and stereodiscrimination did not operate. Conversely, the difluoromethylated $\alpha$-aminonitrile product was obtained with $87 \%$ ee and the trifluoromethylated $\alpha$-aminonitrile was obtained with $94 \%$ ee. The recognition model based on DFT calculations proposed by the au-

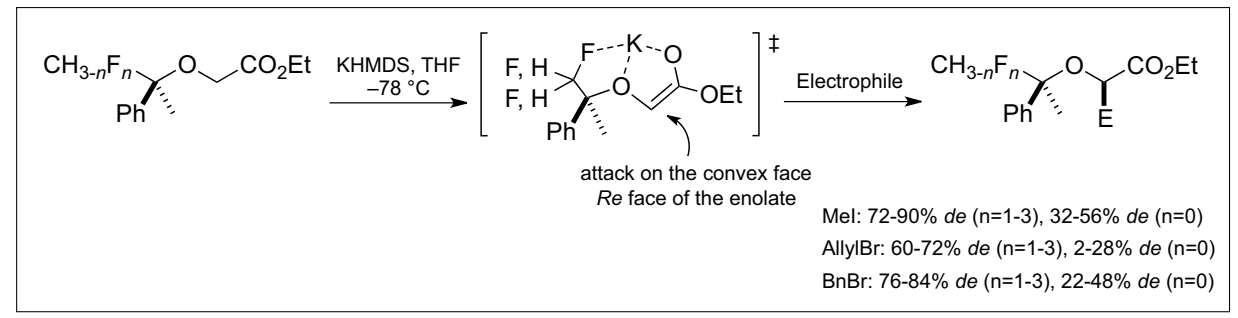

Scheme 2. Assistance of a metal -..fluorine interaction. 


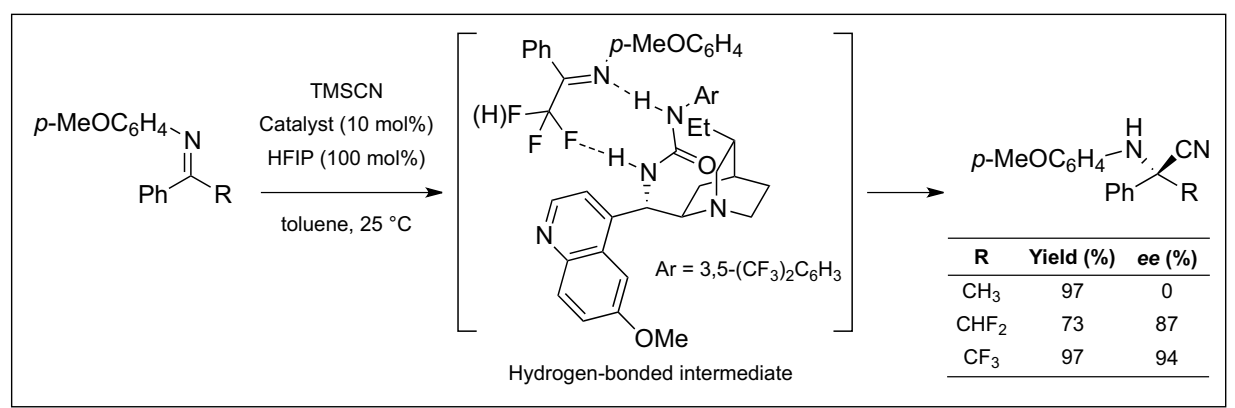

Scheme 3. Hydrogen-bonded complex in the Strecker reaction of fluorinated ketoimines. thors could account for the strong fluorine effect on enantioselectivity observed with fluorinated ketoimines versus the non-fluorinated ketoimine.

\section{An Electron-withdrawing Group Effect}

Fluorine is the most electronegative element with an electronegativity of 3.98 on the Pauling scale. It follows that the strong inductive effect strips electrons from neighbouring atoms with concomitant modification of acid or basic properties. This, indeed, has an impact on the reactivity and stereoselectivity of the fluorinated compounds compared to the non-fluorinated ones. A clear demonstration of the strong electron-withdrawing effect of the $\mathrm{CF}_{3}$ group was given in the chiral Brønsted acid-catalysed arylation of trifluoromethyl ketones (Scheme 4, arylation of 2,2,2-trifluoroacetophenone with indole in the presence of a chiral phosphoric acid). In this example, Ma and co-workers envisioned that: i) the inductive effect of the $\mathrm{CF}_{3}$ group would enhance the reactivity of the substrate versus non-fluorinated acetophenone, ii) the desired FriedelCrafts adduct would be unreactive towards $\mathrm{O}-\mathrm{H}$ group in the adduct; a reaction that occurred when acetophenone was used. In order to validate these hypotheses, DFT calculations were conducted and demonstrated a shortening of the $\mathrm{C}-\mathrm{O}$ bond of the $\mathrm{CF}_{3}$ adduct and significant changes in charge distribution. Consequently, Brønsted acid-catalysed $\mathrm{O}-\mathrm{H}$ activation would be difficult and re-arylation of the $\mathrm{CF}_{3}$-substituted tertiary alcohol might be suppressed (no formation of the bisindole product). Experimental results indicated a much higher reactivity for 2,2,2-trifluoroacetophenone versus acetophenone, and suppression of the re-arylation reaction. Furthermore, excellent enantioselectivities were recorded (Scheme 4). ${ }^{[11,12]}$ In addition, the corresponding difluoromethylphenyl ketone also led to the single Friedel-Crafts adduct with $90 \%$ ee while the monofluoromethylphenyl ketone gave re-arylation thanks to deactivation of the the double Friedel-Crafts adduct because of a lower activation energy of the dehyfluorine atoms determined the structure of the product; two or three fluorine atoms were better than just one to construct fluoromethyl-substituted tertiary alcohols.

\section{$\pi$-Stacking Interactions Influenced by Fluorine Electronic Effects}

The $\pi$-stacking interaction is a non-covalent attractive force between two aromatic rings. This relation can be enhanced using one electron-rich aromatic and one electron-deficient aromatic yielding a strong $\pi$-stacking interaction. This elecdration step. Interestingly, the number of tronic effect has been successfully harnessed in Diels-Alder reactions catalysed by BINOL-derived phosphoric acids. With such kind of chiral Brønsted acids, the 3,3'-substitution of the BINOL moiety is key to reach high stereoinductions. Fluorinated derivatives are frequent and the role of fluorine atom(s) is often to modulate the $\mathrm{pKa}$ of the acid and to bring steric bulk in the case of $\mathrm{CF}_{3}$ group. Furthermore, the propensity of electron-deficient fluorinated phenyl rings to participate in attractive $\pi-\pi$ stacking interactions was demonstrated by Luo and co-workers in (hetero)-Diels-Alder reactions with cyclopentadiene catalysed by a chiral BINOLderived phosphoric acid in the presence of $\mathrm{InBr}_{3} \cdot{ }^{[13]}(E)$-Methyl 2-oxo-4-phenylbut-3-enoate was reacted with cyclopentadiene to afford the hetero-Diels-Alder (HDA) adduct as the major product along with the Diels-Alder (DA) adduct (Scheme 5). Substitution of the remote phenyl moiety of the catalyst with one fluorine atom in para, meta or ortho position led to a gradual increase of enantioselectivity as compared to the non-fluorinated phenyl group, from 80 to $96-98 \% e e$, while no significant change in the HDA/DA ratio was observed. With a pentafluorophenyl group, the enantioselectivity reached $99 \%$ $e e$ for both adducts together with improved

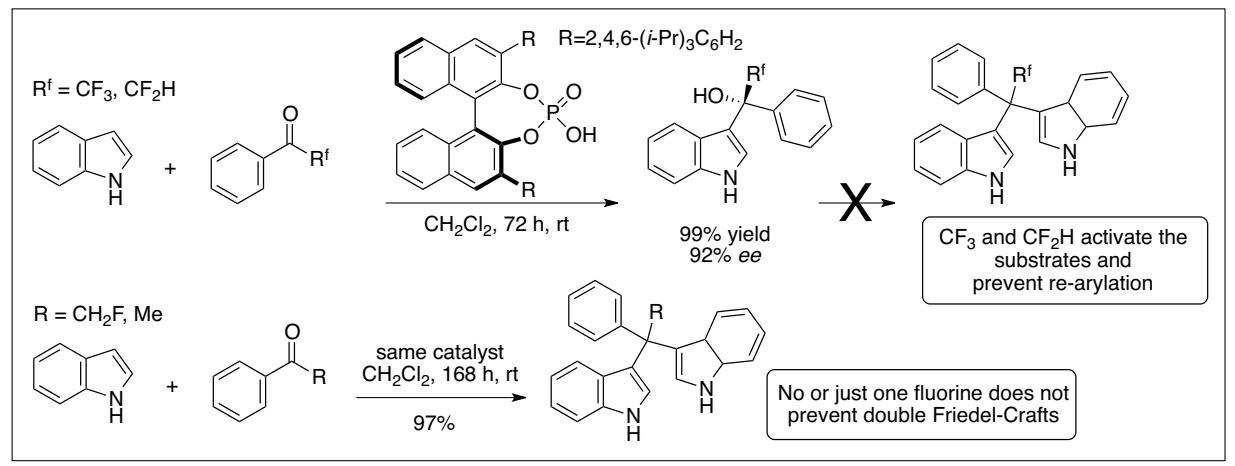

Scheme 4. Double role of the electron-withdrawing $\mathrm{CF}_{3}$ group.

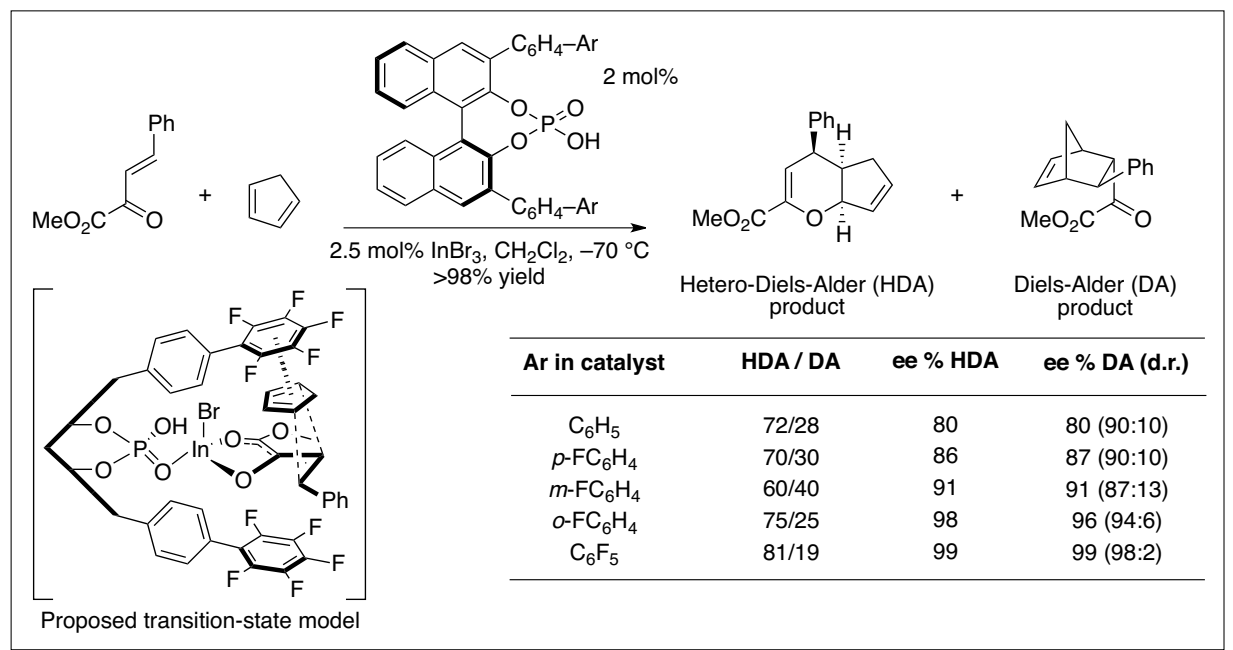

Scheme 5. Stereocontrol in (hetero-)Diels-Alder reactions through $\pi-\pi$ stacking interactions. 
HDA/DA ratio in favour of HDA adduct. The amplitude of the fluorine effect was highly position sensitive and a significant positive stereo effect was obtained with ortho-substitution of the aryl. Moreover, a single ortho-fluorine atom was sufficient to deliver very high stereocontrol similar to that observed with the pentafluorophenyl substitution. It has been suggested that the fluorinated phenyl ring of the catalyst participated favourably to $\pi-\pi$ stacking interactions with the cyclopentadiene leading to adducts in excellent yields and enantiomeric excesses.

\section{Fluorine Stereoelectronic and Electrostatic Effects Induce Privileged Conformations of a Catalyst}

The substitution of hydrogen by fluorine has a profound influence on conformations. Indeed, the fluorine atom is a chemically inert controlling group capable of inducing a high level of conformational organisation by stereoelectronic and electrostatic effects, which are responsible for the gauche effect. It corresponds to the cumulative effects of: i) a stabilising hyperconjugative interaction by orbital alignment of $\sigma_{\mathrm{C}-\mathrm{H}}$ bonding and adjacent low-energy $\sigma_{C-F}^{*}$ antibonding orbitals, ii) an electrostatic interaction $\mathrm{N}^{+} \ldots \mathrm{F}^{\delta^{-}}$. These fluorine effects stabilise privileged conformations and minimise transition-state intermediates. Thus, the wise introduction of a single fluorine atom at a specific position on a catalyst structure can greatly improve the diastereo- and enantiofacial discrimination. Fluorine as a steering element has been investigated in-depth and successfully by the group of Gilmour and also by List, Seebach, and others on many organocatalysts such as proline ${ }^{[14-16]}$ and cinchona alkaloid[17] derivatives, imidazolidinones, ${ }^{[18-20]}$ and $N$-heterocyclic carbenes.[21] Recently, Gilmour and co-workers reported an enantioselective aziridination of enals organocatalysed by fluorinated proline derivatives, in which they pointed out a beneficial fluorine-iminium ion gauche effect (Scheme 6). ${ }^{[22]}$ (S)-2-(fluorodiphenylmethyl)-pyrrolidine was designed for its topology suitable for high expected level of diastereotopic face

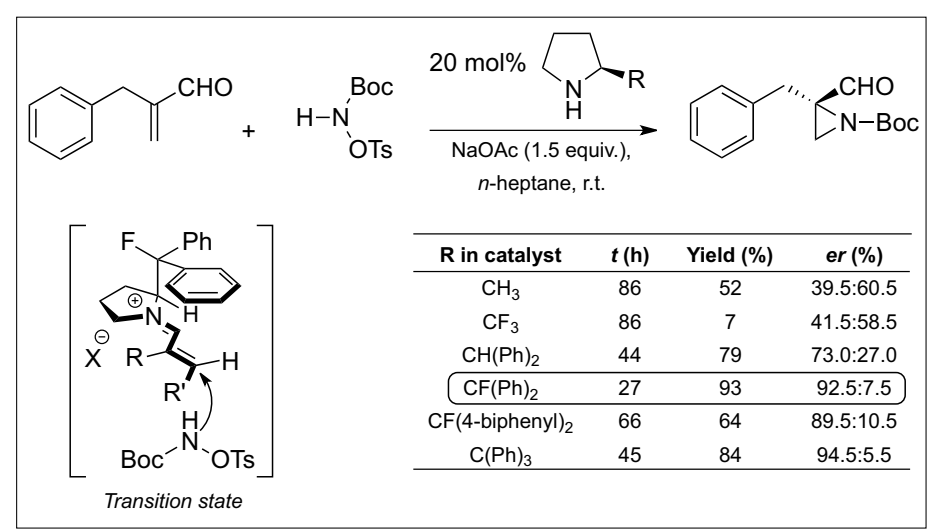

Scheme 6. Fluorineiminium ion gauche effect enhanced the stereochemical outcome of enals aziridination.

selection in the $\beta$-fluoroiminium intermediate formed with the enal in the organocatalysed reaction. The gauche effect positioned the fluorine atom synclinal-endo to the positively charged nitrogen atom thanks to favourable stereoelectronic and electrostatic interactions. With this catalyst $\left(\mathrm{R}=\mathrm{CFPh}_{2}\right)$, high yield $(93 \%)$ and enantioselectivity (92.5:7.5 e.r.) were obtained. To demonstrate the crucial role of the fluorine and the induced gauche effect, the fluorodiphenyl moiety of the catalyst was modified. Erosion of the enantioselectivity was noticed in all cases except for the trityl catalyst that gave an improved level of enantioselectivity but at the expense of a much longer reaction time. This example nicely illustrated the role of fluorine as a control element in catalyst design.

\section{Conclusions}

The strategic installation of fluorine atom(s) to orient the stereodiscrimination is a powerful tool in asymmetric catalysis that certainly merits more attention. In the few examples reported in the literature, the high potential of fluorine to act as a control element was clearly demonstrated. However, the fluorine effect was often examined a posteriori to account for one experimental observation or another. More recently, the fluorine effects, in particular stereoelectronic effects, have been considered a priori for a desired goal. A better understanding and mainstreaming of these effects would be of great asset to any further development of the topic.
[1] T. Liang, C. N. Neumann, T. Ritter, Angew. Chem. Int. Ed. 2013, 52, 8214.

[2] J. Nie, H.-C. Guo, D. Cahard, J.-A. Ma, Chem. Rev. 2011, 111, 455

[3] J.-A. Ma, D. Cahard, Chem. Rev. 2008, 108, PR1

[4] V. Bizet, T. Besset, J.-A. Ma, D. Cahard, Curr Top. Med. Chem. 2014, 14, 901.

[5] D. Cahard, V. Bizet, Chem. Soc. Rev. 2014, 43 , 135.

[6] P. V. Ramachandran, H. C. Brown, ACS Symposium Series 2000, 746, 22.

[7] P. V. Ramachandran, B. Gong, A. V. Teodorović, J. Fluorine Chem. 2007, 128, 844

[8] T. Yamazaki, M. Ando, T. Kitazume, T. Kubota, M. Omura, Org. Lett. 1999, 1, 905.

[9] T. Yamazaki, S. Kawashita, T. Kitazume, T. Kubota, Chem. Eur. J. 2009, 15, 11461

[10] Y.-L. Liu, T.-D. Shi, F. Zhou, X.-L. Zhao, X. Wang, J. Zhou, Org. Lett. 2011, 13, 3826.

[11] J. Nie, G.-W. Zhang, L. Wang, A. Fu, Y. Zheng, J.-A. Ma, Chem. Commun. 2009, 2356.

[12] A. Fu, W. Meng, H. Li, J. Nie, J.-A. Ma, Org. Biomol. Chem. 2014, 12, 1908.

[13] J. Lv, L. Zhang, S. Hu, J.-P. Cheng, S. Luo, Chem. Eur. J. 2012, 18, 799.

[14] C. L. Chandler, B. List, J. Am. Chem. Soc. 2008, 130, 6737.

[15] C. Sparr, W. B. Schweizer, H. M. Senn, R. Gilmour, Angew. Chem. Int. Ed. 2009, 48, 3065.

[16] E.-M. Tanzer, L. E. Zimmer, W. B. Schweizer, R. Gilmour, Chem. Eur. J. 2012, 18, 11334.

[17] E.-M. Tanzer, W. B. Schweizer, M.-O. Ebert, R. Gilmour, Chem. Eur. J. 2012, 18, 2006.

[18] D. Seebach, R. Gilmour, U. Grošelj, G. Deniau, C. Sparr, M.-O. Ebert, A. K. Beck, L. B. McCusker, D. Šišak, T. Uchimaru, Helv. Chim. Acta 2010, 93, 603

[19] U. Grošelj, Č. Podlipnik, J. Bezenšek, J. Svete, B. Stanovnik, D. Seebach, Helv. Chim. Acta 2013, 96,1815

[20] C. Sparr, R. Gilmour, Angew. Chem. Int. Ed. 2010, 49, 6520.

[21] Y. P. Rey, R. Gilmour, Beilstein J. Org. Chem. 2013, 9, 2812.

[22] I. G. Molnár, E.-M. Tanzer, C. Daniliuc, R. Gilmour, Chem. Eur. J. 2014, 20, 794. 